\title{
Unusually low density regions in the compressed slow wind: Solar wind transients of small coronal hole origin
}

\author{
Yong C.-M. Liu ${ }^{1}$, Zhaohui $\mathrm{Qi}^{1,2}$, Jia Huang ${ }^{3}$, Chi Wang ${ }^{1,2}$, Hui Fu ${ }^{4}$, Berndt Klecker ${ }^{5}$, \\ Linghua Wang ${ }^{6}$, and Charles J. Farrugia ${ }^{7}$
}

\footnotetext{
${ }^{1}$ State Key Laboratory of Space Weather, National Space Science Center, Chinese Academy of Sciences, Beijing, PR China e-mail: liuyong@spaceweather.ac.cn

2 College of Earth Sciences, University of Chinese Academy of Sciences, Beijing, PR China

3 Climate and Space Sciences and Engineering, University of Michigan, Ann Arbor, MI 48109, USA

${ }^{4}$ Shandong Provincial Key Laboratory of Optical Astronomy and Solar-Terrestrial Environment, Institute of Space Sciences, Shandong University, Weihai 264209, Shandong, PR China

5 Max-Planck-Institut für extraterrestrische Physik, Garching, Germany

${ }^{6}$ School of Earth and Space Sciences, Peking University, 100871 Beijing, PR China

7 Institute for the Study of Earth, Oceans and Space, University of New Hampshire, Durham, New Hampshire, USA
}

Received 14 May 2019 / Accepted 2 January 2020

\begin{abstract}
We report on two small solar wind transients embedded in the corotating interaction region, characterized by surprisingly lower proton density compared with their surrounding regions. In addition to lower density, these two small solar wind transients showed other interesting features like higher proton temperature, higher alpha-proton ratios, and lower charge states $\left(\mathrm{C}^{+6} / \mathrm{C}^{+5}\right.$ and $\left.\mathrm{O}^{+7} / \mathrm{O}^{+6}\right)$. A synthesized picture for event One combining the observations by STEREO B, ACE, and Wind showed that this small solar transient has an independent magnetic field. Back-mapping links the origin of the small solar transient to a small coronal hole on the surface of the Sun. Considering these special features and the back-mapping, we conclude that such small solar wind transients may have originated from a small coronal hole at low latitudes.
\end{abstract}

Key words. Sun: heliosphere - solar wind - magnetic reconnection - Sun: oscillations

\section{Introduction}

Interplanetary space is populated by solar wind plasma which consists of protons, electrons, and other ion species with low abundances like alpha particles and heavier ions. They are all emitted radially from the rotating Sun and drag the magnetic field lines out into a spiral configuration of the Parker spiral). Earlier studies have classified the solar wind into fast and slow types mainly according to their speed and other physical properties (McComas et al. 2000; von Steiger et al. 2000; Schwenn 2006; Zurbuchen et al. 2012). In general, the fast solar wind has higher proton temperature, lower density, lower charge state ratios $\left(\mathrm{O}^{7+} / \mathrm{O}^{6+}, \mathrm{C}^{6+} / \mathrm{C}^{5+}\right)$, higher alpha-to-proton ratio, and lower elemental abundance ratios $(\mathrm{Fe} / \mathrm{O})$ compared to the slow solar wind (Geiss et al. 1995; Feldman et al. 2005; Xu \& Borovsky 2015; Wang 2016). Zhao et al. (2009) carried out statistical studies on the properties of the solar wind at solar minimum and also showed that a very small portion of low speed wind exhibits a composition similar to the fast wind. Feldman et al. (2005) suggested a similar idea using solar images by Solar and Heliospheric Observatory (SOHO). The region where the fast solar wind interacts with the slow wind is called a stream interaction region (SIR) or co-rotating interaction region (CIR) if it persists over a solar rotation (Gosling 1996; Jian et al. 2006; Broiles et al. 2012). The fast wind compresses the leading slow wind. In the compression region, the density is usually higher than in the undisturbed slow wind ahead. Therefore, the stream interface (SI) between the fast and slow wind is characterized by a rise in temperature, a decrease in proton density, and a compression of the magnetic field (Gosling et al. 1978, 1993; Jian et al. 2006; Simunac et al. 2009). Because the stream interface separates different solar wind originates from different regions, which has different freeze-in temperatures, abundance ratios like alphato-proton and ionic charge state ratios like iron-to-oxygen will thus change at the interface (Gosling et al. 1978; Borrini et al. 1981; Wimmer-Schweingruber et al. 1997; Crooker et al. 1999; Jian et al. 2019).

The fast and slow winds usually do not mingle because they have different solar origins. Fast solar wind originates from coronal hole regions with open magnetic field lines (Zirker 1977; Bame et al. 1993; McComas et al. 2002; Fu et al. 2015), while slow wind originates from the vicinity of the streamer belt region (Feldman et al. 2005; Abbo et al. 2016; He et al. 2009). Some recent statistical studies show that a small portion of slow wind has fast solar wind composition and charge states (Zhao et al. 2014) although no exact time range was listed for these unusual slow winds. Other recent studies suggest that fast and slow wind should instead be called, respectively, "coronal hole wind" and "streamer belt wind" (Xu \& Borovsky 2015). We still use the denomination fast and slow wind to avoid confusion, noting that some solar wind may have fast-wind like composition but low speed. Bruno \& Telloni (2015) studied the fluctuations in the slow wind and concluded that there are two types of slow wind, one with more Alfvénic fluctuations originating from the coronal hole boundary, and the other with less Alfvénicity originating from active regions or streamer belt. Recently, D'Amicis et al. (2019) 
Table 1. Event One time period for spacecraft STEREO B, Wind, ACE, and STEREO A.

\begin{tabular}{lccc}
\hline \hline Spacecraft & Time period studied & Low density region time & Stream interface \\
\hline STEREO B & $04 / 08$ 2030-04/09 1630 & $04 / 090632-04 / 090837$ & $04 / 091014$ \\
Wind & $04 / 081530-04 / 091130$ & $04 / 090102-04 / 090349$ & $04 / 090557$ \\
ACE & $04 / 081530-04 / 091130$ & $04 / 090035-04 / 090323$ & $04 / 090537$ \\
STEREO A & $04 / 081000-04 / 090600$ & N/A & $04 / 082056$ \\
\hline
\end{tabular}

Notes. All times are in the format MM/DD HHMM.

found that Alfvénic slow winds have higher charge state ratios similar to fast winds during solar maximum, suggesting that they originate from a coronal hole. Another study by Wang et al. (2019) traced most of these Alfvénic solar winds back to active regions.

Small transients are common in the solar wind (Yu et al. 2016), and a subset of them are small flux ropes that show twisted magnetic structures (Moldwin et al. 2000; Huang et al. 2017). In general, small transients have short duration, decreased magnetic field variability, and low proton temperature and/or proton beta (Kilpua et al. 2009). Furthermore, Yu et al. (2014, 2016) used additional parameters, such as enhanced magnetic field strength and low Alfvén Mach number, to characterize small transients. Their statistical studies suggest that more than $80 \%$ of small transients occur in the slow solar wind, and small transients are more frequently observed during solar minimum. In order to understand the source regions and origin mechanisms of small transients, some researchers use in situ measurements (Janvier et al. 2014; Yu et al. 2016; Huang et al. 2018), and some researchers use imaging observations to trace back transients entrained in CIRs (Rouillard et al. 2009).

Although some transients can be traced back to CIRs, the origins of most small solar transients are still puzzling. We report two small solar wind transients of low density embedded in the compressed slow wind in a CIR. Both of them are characterized by some features observed in fast solar wind, like lower density and lower oxygen charge states, and we link their origins to small coronal holes at lower latitudes.

The paper is organized as follows. The instruments and data are presented in Sect. 2. In situ Observations by STEREO, ACE, and Wind for events One and Two are described in Sect. 3. In Sect. 4 we combine the observations of three spacecraft to determine the boundary of the low density region; the back-mapping is also presented to determine its origin. The origin of such a solar wind transient is discussed in Sect. 5 and conclusions are drawn in Sect. 6.

\section{Data}

The data used in this study were obtained from the Wind, Advanced Composition Explorer (ACE), and Solar TErrestrial RElations Observatory (STEREO)-A/B spacecraft. The Wind spacecraft was launched in 1994, and after orbiting Earth's magnetosphere for years it was placed in a halo orbit around the L1 Lagrange point in early 2004 to observe the unperturbed solar wind. The Wind solar wind plasma data, including electrons, proton, and alpha particles, are obtained from two instruments: the plasma and suprathermal electron data from the 3D Plasma and Energetic Particles Experiment (3DP) (Lin et al. 1995) and the magnetic field data from the Magnetic Field Investigation (MFI) (Lepping et al. 1995). The 3DP and MFI data we used both have a three-second time resolution. To make a comparison with the measurements from the 3DP instrument, the Solar
Wind Experiment (SWE) (Ogilvie et al. 1995) ion data with the resolution of 92 s were also used.

ACE was launched in mid-1997 (Stone et al. 1998) and orbits around the Sun-Earth L1 libration point. The data we used are from the Solar Wind Electron Proton Alpha Monitor (SWEPAM) (McComas et al. 1998) measuring protons and $\alpha$ particles, the Solar Wind Ionization and Composition Spectrometer (SWICS) (Gloeckler et al. 1992) measuring solar wind ion compositions and charge states, and the magnetometer (MAG) (Smith et al. 1998). The ACE composition data we used have a one-hour time resolution.

The twin STEREO spacecraft were launched in late 2006. Both of them are orbiting the Sun near 1 AU with STEREO A ahead of the Earth and STEREO B behind it. The longitudinal separation between STEREO A and B increases by approximately $45^{\circ}$ per year (Kaiser et al. 2008). The two spacecraft are equipped with identical instruments. The data we used are obtained from the Plasma and Suprathermal Ion Composition (PLASTIC) (Galvin et al. 2008) and the In situ Measurements of Particles And CME Transients (IMPACT; Luhmann et al. 2008) investigations. The former provides the $1 \mathrm{~min}$ average properties of the solar wind calculated from 1D Maxwellian fits. The latter consists of seven instruments, but we used data from only two of them, namely the Magnetometer (MAG; Acuña et al. 2008) and Solar Wind Electron Analyzer (SWEA; Sauvaud et al. 2008) instruments.

\section{Observations}

We present two events of low density small solar wind transients in the compressed region in this section. Both of them have features suggesting that they may have a coronal hole origin despite their low speed.

\subsection{Event One}

Event One was observed around 8-9 April 2007 by STEREO B, Wind, and ACE. The time period we investigated for each spacecraft is listed in Table 1. Figure 1a shows the locations of STEREO A and B, ACE, and Wind in the ecliptic plane ( $X Y$ plane in GSE coordinates), and the heliospheric longitude and latitude (HCI coordinates) of the spacecraft are shown in Fig. 1b. In the Geocentric Solar Ecliptic (GSE) coodinate system, the positive $X$ is along the Earth-Sun line towards Sun, $Z$ is the ecliptic north pole, and $Y$ is the cross product of $Z$ and $X$. In Heliocentric Inertial (HCI) coordinate system, the positive $X$ is the solar ascending node in the ecliptic plane while $Z$ is the solar rotation axis, $Y$ is the cross product of $Z$ and $X$. The locations and coordinates of the spacecraft are obtained from the Satellite Situation Center Web (SSCWeb) ${ }^{1}$ and STEREO Science Center ${ }^{2}$.

\footnotetext{
1 http://sscweb.gsfc.nasa.gov

2 https://stereo-ssc.nascom.nasa.gov/where. shtml
} 


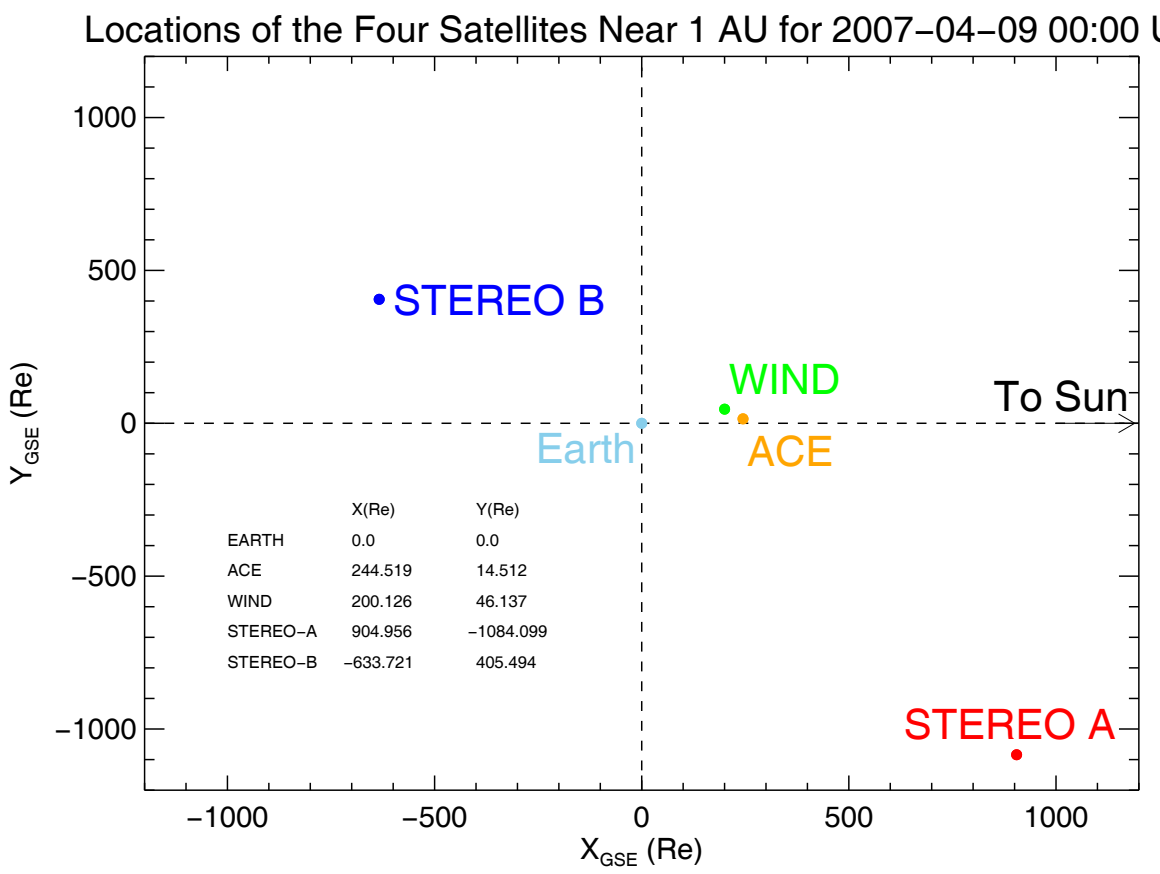

(a)

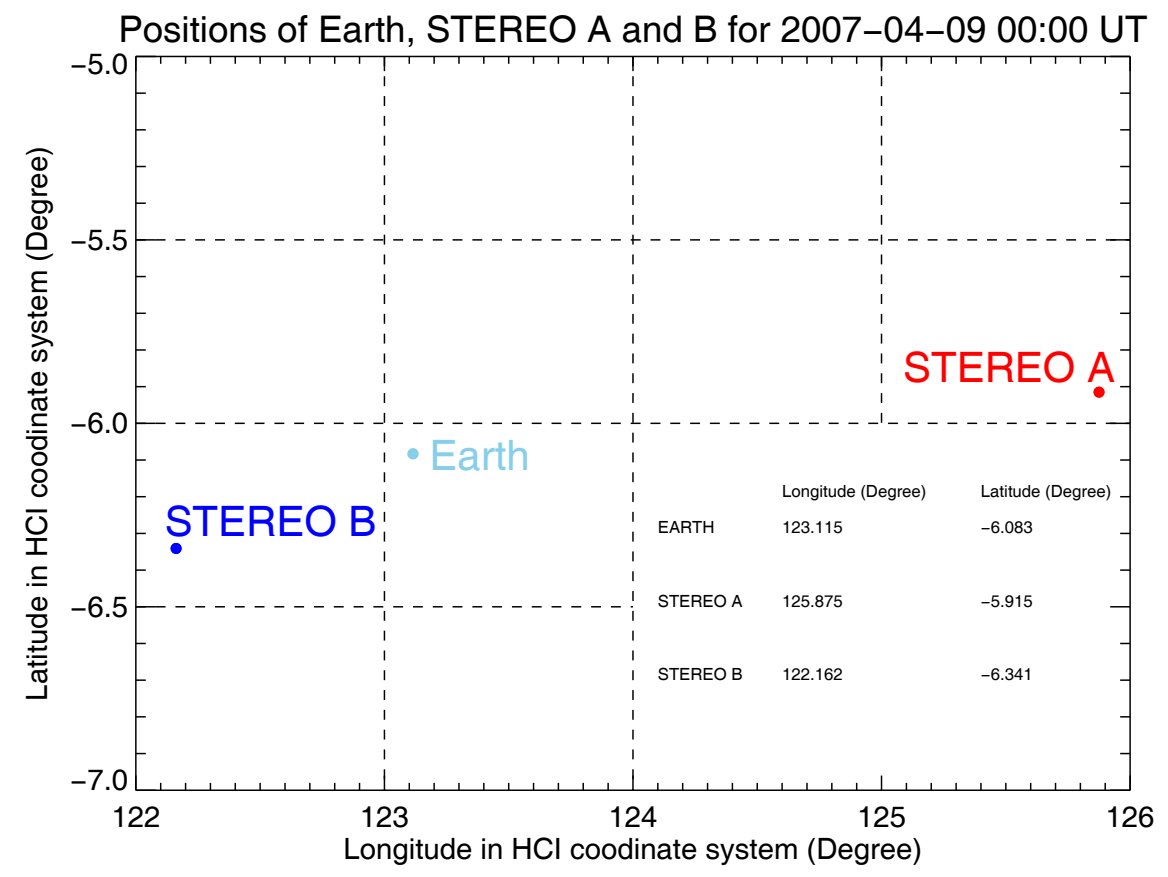

(b)

Fig. 1. (a) Locations of the spacecraft STEREO A/B, ACE, and Wind in GSE coordinates. (b) Heliospheric longitude and latitude of the Earth and the spacecraft STEREO A/B in HCI coordinates. Both data are obtained on 9 April 2007, 00:00 UT.

On 9 April 2007, ACE and Wind were very close to Earth while the STEREO spacecraft were separated from Earth by less than $3^{\circ}$ in heliospheric longitude. The heliospheric latitudinal separation was much smaller than that of heliospheric longitude (see Fig. 1b); therefore, all spacecraft were approximately located in the ecliptic plane.

Figure 2 shows a CIR observed by STEREO B on 9 April 2007. From top to bottom the panels show the proton number density, proton bulk speed, observed and expected proton temperature, suprathermal electron pitch angle distributions, magnetic field magnitude, magnetic field components, magnetic field azimuthal angle $\phi_{B}$, and alpha-to-proton number density ratio. It has been known for decades that there is a clear correlation between solar wind velocity and proton temperature (Lopez \& Freeman 1986; Neugebauer et al. 2003). The $\phi$ angle is magnetic field azimuthal angle between the $B_{R}$ and $B_{T}$ components in RTN coordinate systems (Borrini et al. 1981; Broiles et al. 2012). The Parker spiral angle is $135^{\circ}$ (away from the Sun) and $315^{\circ}$ (toward to the Sun), two values due to the tendency of the solar wind magnetic field to align along an Archimedean spiral while corotating with the Sun (Parker 1958; Borovsky 2010). The expected temperature is based on the statistical relation of the proton temperature versus the solar wind speed (Elliott et al. 2005; Yu et al. 2014). One of the criteria used to identify interplanetary coronal mass ejections (ICMEs) is that the observed temperature is lower than half the expected temperature.

The SI passed over the STEREO B spacecraft at about 10:14 UT on 9 April 2007, as shown by the black vertical line in Fig. 2. The interface is characterized by a simultaneous sharp 


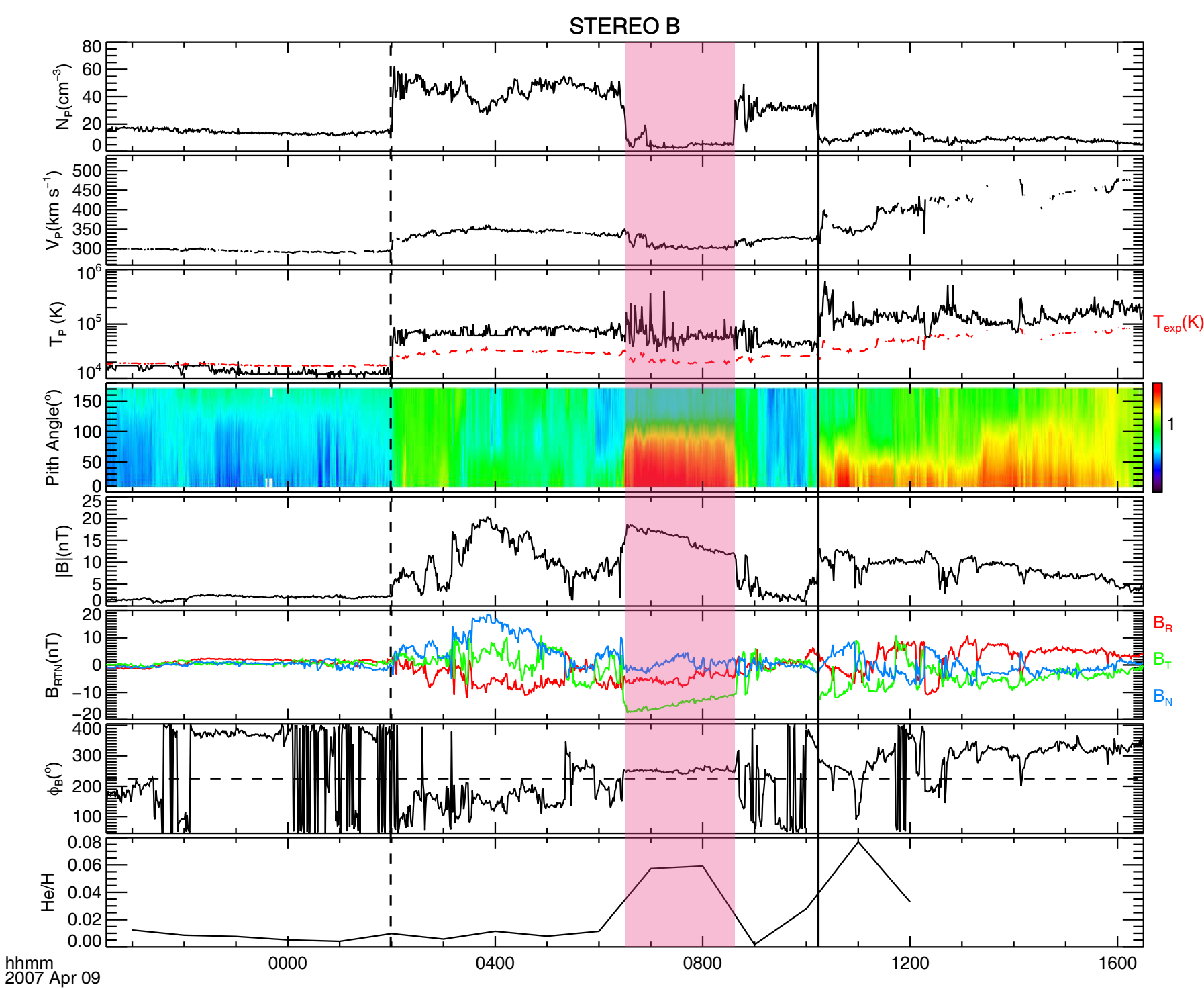

Fig. 2. In situ solar wind plasma parameters observed by STEREO-B. From top to bottom: proton density, proton bulk velocity, proton temperature and expected temperature (red dashed), pitch angle distribution of $\sim 272 \mathrm{eV}$ suprathermal electrons, magnitude of magnetic field, components of the magnetic field in RTN coordinates, magnetic field azimuthal angle (225 ; dashed horizontal line), alpha-to-proton number ratio. The solid vertical line indicates the stream interface. The dashed line indicates the shock in front of the CIR. The pink shading shows the very low density region.

drop in proton density and a rise in proton temperature and proton bulk speed. This SI is also included in the list made by Lavraud et al. (2010). The dashed line indicates the shock in front of the SI. The compressed slow wind between the SI and the shock is usually of higher density. However, the solar wind transient, the region marked with pink shading in the figure, has a very low proton density $\left(\sim 2 \mathrm{~cm}^{-3}\right)$, which is much lower than that of the surrounding compressed slow wind $\left(\sim 40 \mathrm{~cm}^{-3}\right)$ and the uncompressed slow wind $\left(\sim 15 \mathrm{~cm}^{-3}\right)$ far ahead. It is even slightly lower than that of the fast wind trailing the CIR. In addition to the strikingly low proton density, the flux of suprathermal electrons increases sharply compared to the neighboring compressed slow wind. The alpha-to-proton number ratio $(\mathrm{He} / \mathrm{H})$ within the region also increases from $2 \%$ to nearly $6 \%$. The proton temperature within this region stays the same except for several short spike-like increases. The magnetic field has a nearly constant $\phi$ angle approximately equal to $225^{\circ}$, which suggests that the magnetic field is oriented nearly perpendicular to the
Parker spiral. The suprathermal electrons have two distinctive features: they flow along the magnetic field line towards the Sun, which suggests that the magnetic field lines roll back from its usual configuration (Owens et al. 2013; Liu et al. 2014), and they have higher flux in pitch angles from $0^{\circ}$ to $90^{\circ}$, which suggests that electrons have gone through strong scattering. The rolling back may help to explain the direction of magnetic field lines. Except for the temperature, all these features suggest that the small solar wind transient is distinct from its surroundings and may have a different origin from them.

The SI passed over the Wind spacecraft at about 06:00 UT on 9 April 2007, as shown in Fig. 3. The solar transient is also observed in front of the SI, embedded in the compressed slow wind. The proton density is about $4 \mathrm{~cm}^{-3}$, which is significantly lower than the density of the slow wind in the surrounding area. The flux of suprathermal electrons is also higher. The alpha-toproton number ratio, although variable, is significantly higher than that of the slow wind in the neighboring region. The proton 


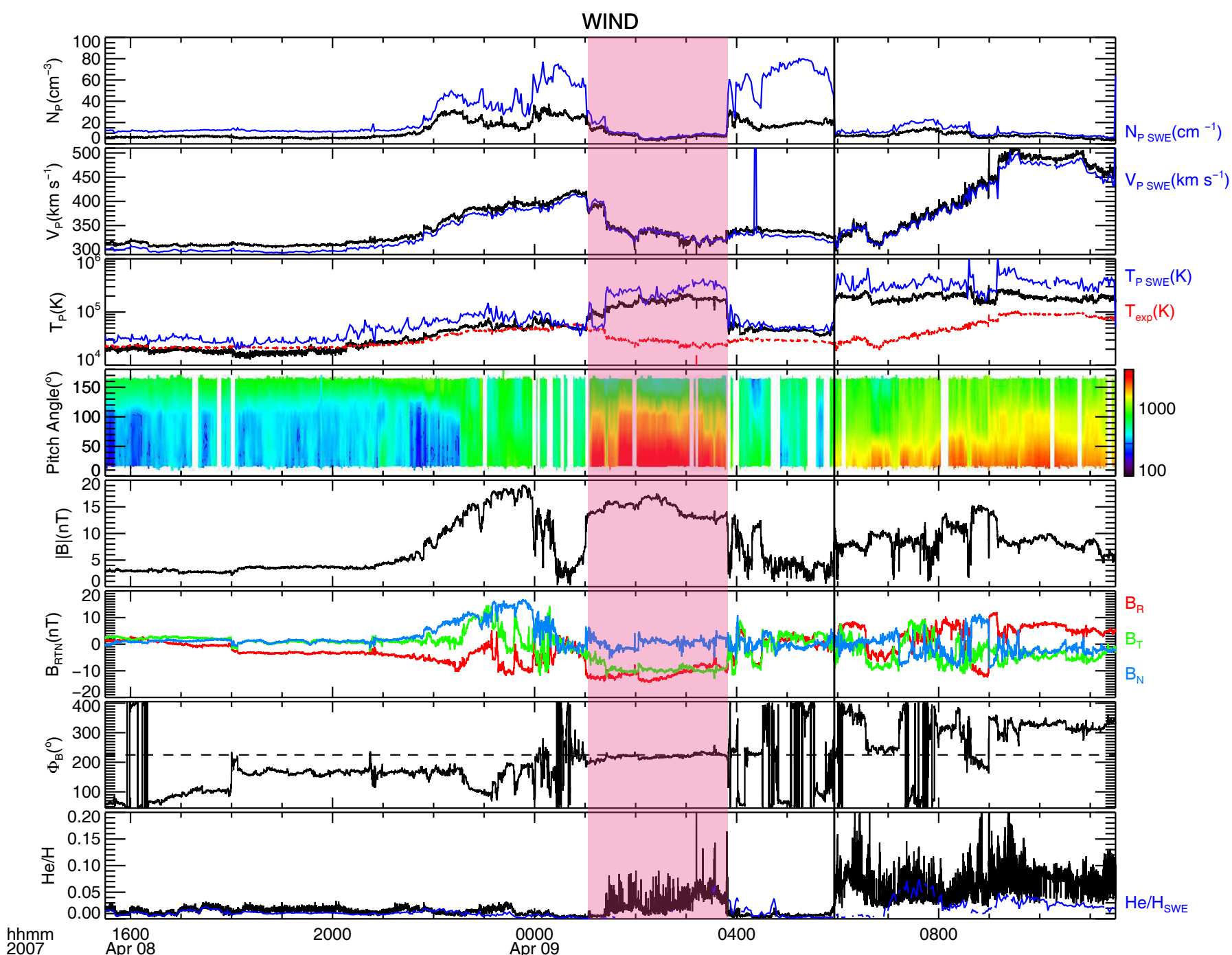

Fig. 3. In situ plasma and magnetic field observations by Wind in the same format as Fig. 2. For panels 1, 2, 3, and 8 the black lines are the data from 3DP, while the blue lines are from SWE.

temperature is notably higher within the low density region. Although they are observing the same plasma parcel, it is puzzling that the temperature observed by STEREO B is not significantly higher than that of the surrounding region except for the spikes. To make a comparison with the measurment from 3DP, we overplot the hydrogen and helium measurements from the SWE instrument on Wind in Fig. 3 (in blue). The results show consistently that the proton density is lower, while the temperature is higher within the pink region.

We examined other features of this small solar transient. The magnitude of the magnetic field decreases with a nearly constant rate and the $\phi$ angle has a very small variation at STEREO B; however, this constant decrease is not clear in Wind or ACE. The field, although very variable, is mainly parallel to the ecliptic plane (see the small $\mathrm{Bn}$ in the sixth panel of Figs. 2 and 3) and nearly perpendicular to the Parker spiral. This is a pressure-balanced structure (PBS), as shown in bottom panel of Fig. 4, where all pressures are plotted. Thus, the mean total pressure value of this low density region, which includes alphas, protons, electrons, and magnetic pressure, is $0.14 \pm 0.01 \mathrm{nPa}$. The structure is bounded by two nearly parallel tangential discontinuities. Burlaga et al. (1990) reported a few PBS events based on Voyager observations, showing that the temperature, density, or magnetic field may vary within the structure, but that the total pressure remains constant. The events they presented did not have sharp changes at the boundaries of the structure and our observations show that such PBSs may have sharp boundaries.

Figure 5 shows the passage of the structure at ACE. The proton density of $12 \mathrm{~min}$ resolution is plotted as the red line in the first panel in addition to the 1 min proton density, because the proton density is not available for most of this time period. The charge states ratios are also plotted for heavier ions like carbon and oxygen. We omitted the $\mathrm{He} / \mathrm{H}$ ratio since it is quite similar to that of the Wind observations. During the highlighted period, the ACE data show similar features, such as lower proton density, higher proton temperature, increased suprathermal electron flux, and smoothly rotating magnetic field, consistent with the observed low density region by STEREO B, although STEREO B observed a shock leading the CIR.

Figure 6 shows the solar wind observations by STEREO A near the SI before STEREO B, Wind, and ACE observed the low density region. The SI passed the spacecraft at 21:00 UT April 8 2007. However, there is no trace of a low density region ahead of the SI as observed by the other spacecraft. Evidently, STEREO A did not observe this feature. 


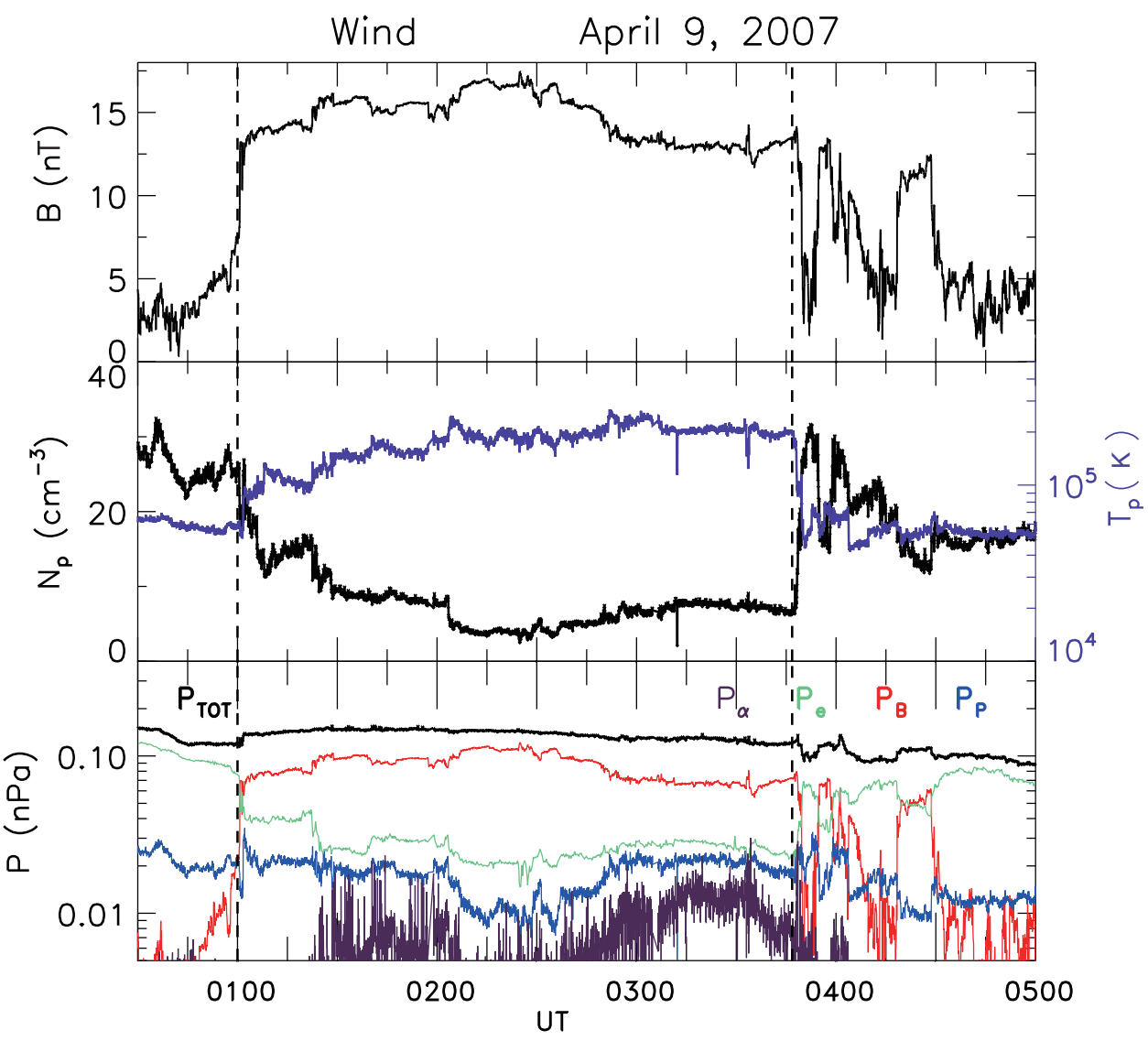

Fig. 4. For a short period: total magnetic field, number density, proton temperature, and pressure due to $\alpha$ particles (purple), electrons (green), magnetic field (red), and protons (blue). Also shown is the total pressure (black).

\subsection{Event Two}

The second low density solar wind transient leading a stream interface was observed by ACE on 7 June 2011, as shown in Fig. 7a, plotted in the same format as Fig. 5. The time period we investigate for each spacecraft is listed in Table 2. From the table, the twin spacecraft of STEREO did not observe this parcel of solar wind with lower density since both of them are separated from ACE/Wind by at least $90^{\circ}$ in heliospheric longitude during this time period. The pink region in the figure denotes the region with low density of about $4.6 \mathrm{~cm}^{-3}$, lower than the surrounding wind with density varying around $13.7 \mathrm{~cm}^{-3}$, and also similar to the uncompressed slow wind leading the shock indicated by the vertical black dashed line in Fig. 7. The flux of suprathermal electrons is also enhanced compared to the surrounding region. The oxygen charge state ratio $\mathrm{O}^{7+} / \mathrm{O}^{6+}$ is about 0.15 , which is lower than the surrounding slow wind. The azimuthal angle of magnetic field changes at a constant rate. Although not higher than the expected temperature, the observed proton temperature in the region is not significantly different from that of the uncompressed slow wind. Except for the comparison with the $T_{\exp }$, all other features (lower density, lower oxygen charge states, and increased suprathermal electron flux) are consistent with those in event One.

Contrary to event One, there is a heliospheric current sheet (HCS) crossing outside the low density region on 11 June 2011, 14:00 UT in event Two (see Fig. 7b, vertical red dashed line). In event One the position of HCS crossing is near the SI, but the accurate postion is hard to confirm because of the departure of HCS and the True Sector Boundary (TSB; Huang et al. 2016a). The HCS is defined as a sustained change in signs of the magnetic field; it is accepted as a separation for the different polarity of the interplanetary magnetic field (Crooker et al.
2004; Liu et al. 2014) when it is consistent with TSB, which is defined as the change in direction of PADs (Kahler et al. 1996; Foullon et al. 2009). When the magnetic field lines roll back, HCS is separated from TSB (Owens et al. 2013; Huang et al. 2017). Thus, the low density region solar wind does not depend on the streamer belt structure or on HCS.

\section{Analysis}

A multi-spacecraft analysis of the low density region for event One is presented in this section along with the back-mapping to the solar source surface for both events.

\subsection{Synthesized picture}

To understand the spatial structure of the small solar wind transient, a synthesized picture combining the STEREO B and Wind observations is presented in Fig. 8. The picture shows the spatial distribution of solar wind when the stream interface passed STEREO B. The Wind observation is shifted to this time. We assume that the solar wind is moving at a constant speed; therefore, the spatial scale for the solar wind can be determined based on the time duration and solar wind speed. The displacement of the spacecraft is negligible compared with the solar wind movement during this time period. The solar wind observed during this time is divided into four parts: the leading slow wind, the low density wind, the trailing slow wind, and the fast wind.

The magnetic field lines are plotted as black curves with blue arrows showing the directions; the suprathermal electron flow is represented by the red arrows and semicircles. The red arrow and semicircle along the magnetic field direction represent the direction and distribution of suprathermal electrons, while the arrow 


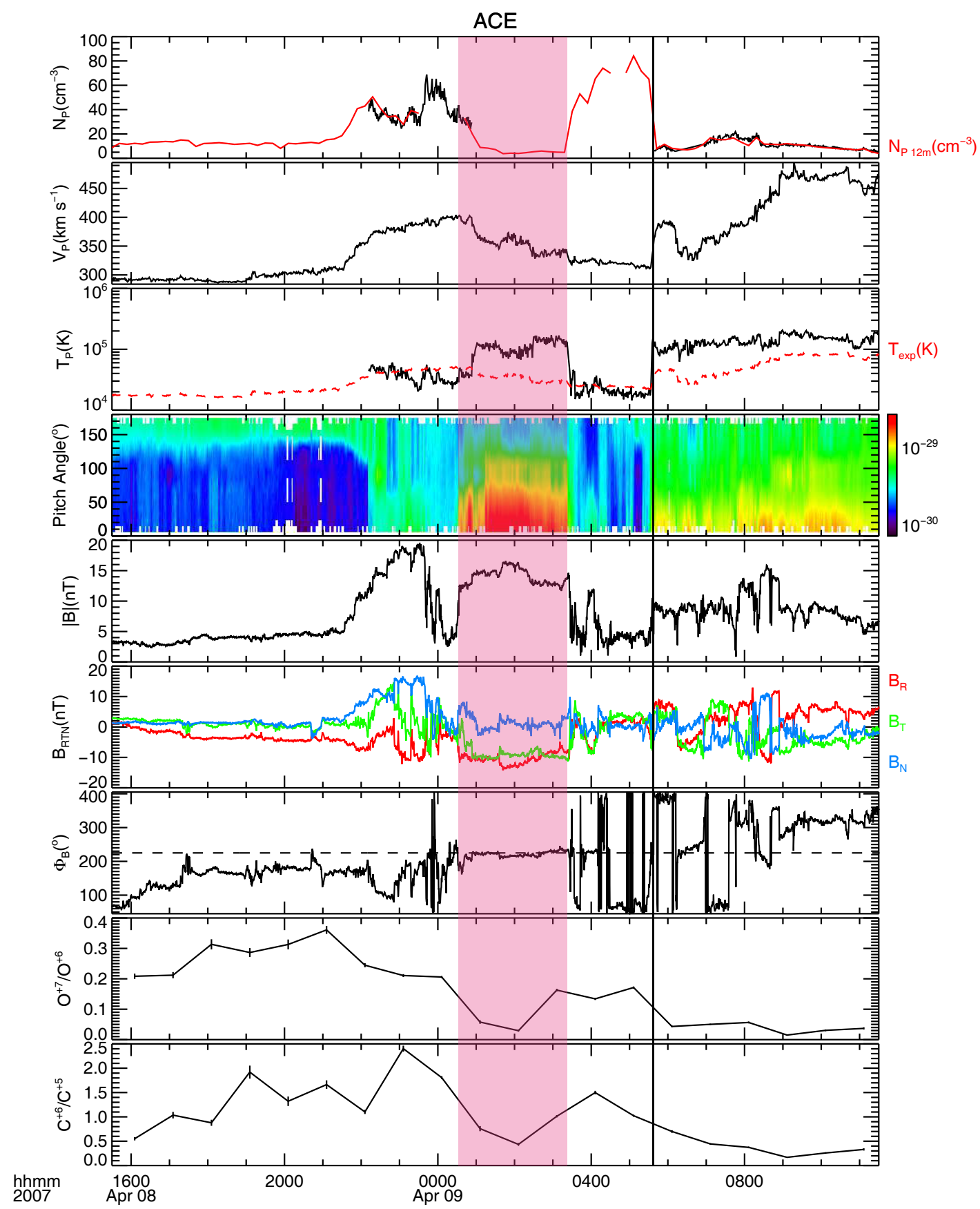

Fig. 5. In situ data observed by ACE. From top to bottom: proton density, proton bulk speed, proton temperature, pitch angle distribution of $\sim 272 \mathrm{eV}$ electrons, magnitude, components and $\phi$ angle of the magnetic field, and the $\mathrm{O}^{7+} / \mathrm{O}^{6+}$ and $\mathrm{C}^{6+} / \mathrm{C}^{5+}$ charge state ratios of oxygen and carbon. The proton density is combined with $1 \mathrm{~min}$ resolution data (black line) and 12 min resolution data (red). The pink shaded bar in the low density region and the solid line represents the interface, as in Fig. 2.

indicates a narrow electron strahl and a semicircle represents an azimuth spread of nearly $90^{\circ}$. The boundary of the low density region is determined by connecting the end points as observed by Wind and STEREO B. The boundary is nearly parallel to the magnetic field lines within the region. The low density region has a distinct magnetic structure independent from the surrounding solar wind. The extension in the $X$ direction of the low density region is approximately a few hundred Earth radii, and it is wider at smaller $Y$, as observed by Wind. In the $Y$ direction, the low density region may extend in both positive and negative $Y$ along the boundary, as shown by the dashed lines extending from the boundary. The shape of the boundary suggests that the solar transient has a narrow ribbon-like shape, corresponding to a flow sustained for a certain time period. The longitudinal separations between the spacecraft are much larger than the latitudinal separations. It is more likely that the extension in the negative $Y$ direction might be limited so that STEREO A did not observe the feature, although we cannot exclude the possibility that it is caused by limited $Z$ extension.

\subsection{Origin from the Sun}

In order to help understand the origin of the small solar transient, the synoptic map from the Global Oscillation Network Group 


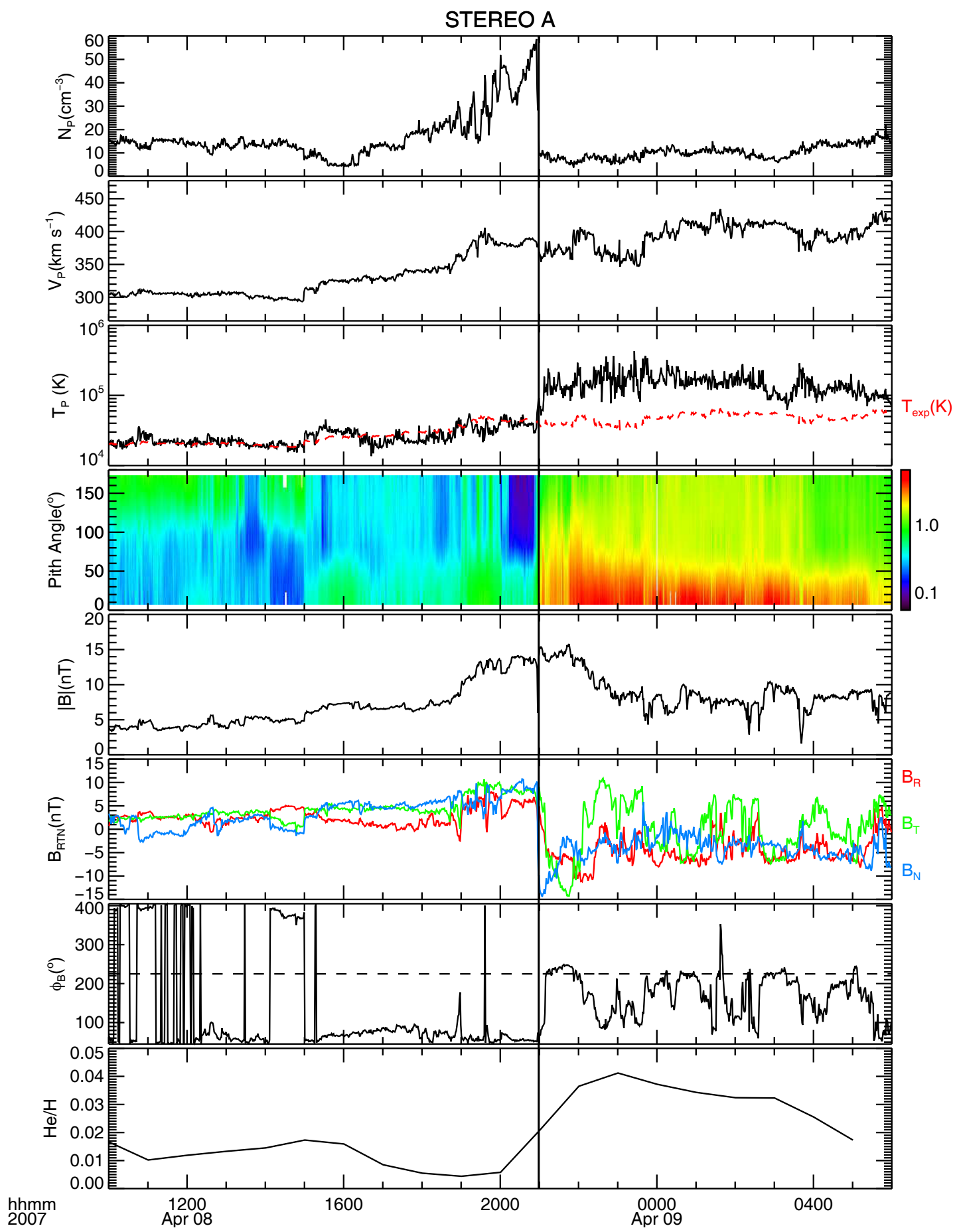

Fig. 6. In situ data from STEREO-A. The panels are in the same format as STEREO B. STEREO A did not encounter the low density region; only the SI is evident (vertical line).

(GONG) is presented in Fig. 9 (courtesy of GONG), together with the back-mapping of the in situ observations in Fig. 10. It shows the result of the PFSS model of CR 2055 with the timeline going from right to left. The red (green) shaded region indicates the negative (positive) polarity coronal holes mainly distributed in the high latitude region with some small patch-like coronal holes distributed at lower latitudes. The horizontal green and blue line beneath the equator indicates the projected trajectories of Earth and STEREO B. In event One their latitudinal difference is so small that their trajectories almost overlap. In event Two, because STEREO A/B did not observe the low density region, we only project the Earth trajectories on the map. The vertical black solid line is the approximate position of the low density region traced back to the source surface, there should be six solid lines and four solid lines (forward and reverse boundary of the low density for each event) in event One and Two, respectively, we just show the minimum and maximum longitude of the six lines for clarity. This back-mapping is based on the average speed of the low density region. The low density region observed by STEREO B, Wind, and ACE is near the equator. Figure 9 shows that these two events may originate from the closed magnetic field region. 


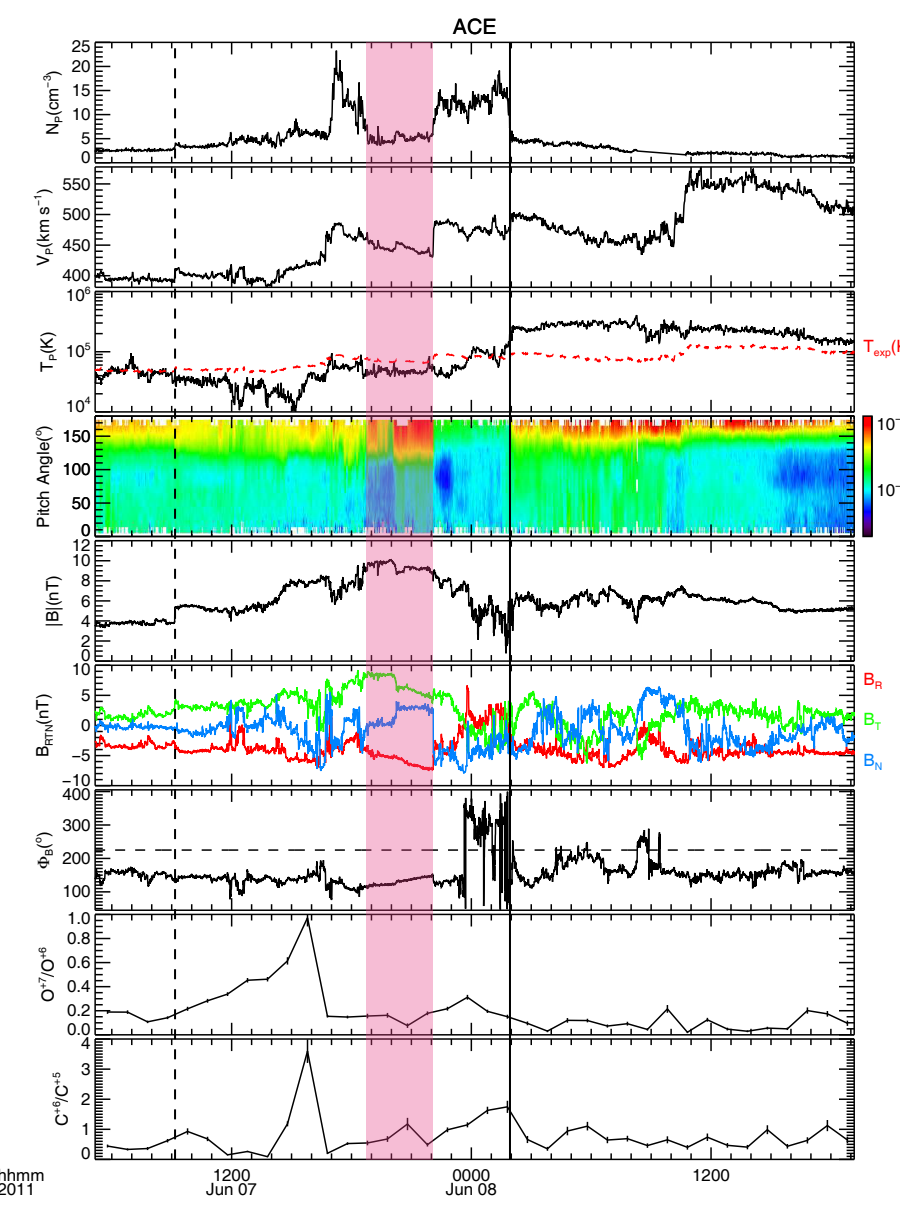

(a)

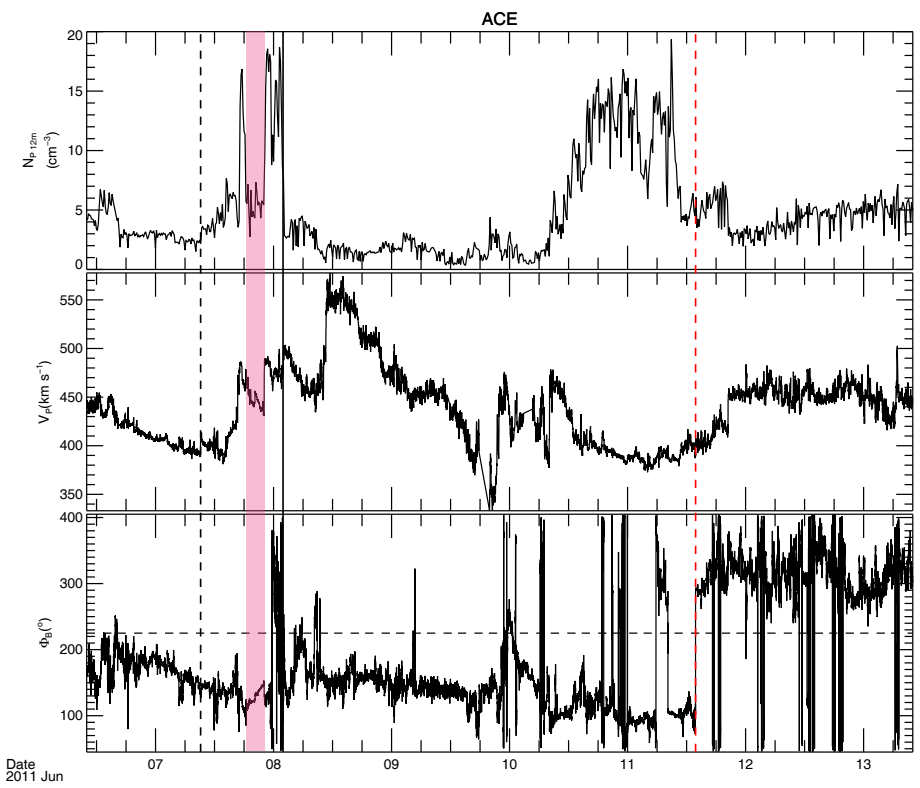

(b)

Fig. 7. (a) ACE observations for event Two. From top to bottom: proton density, proton bulk speed, proton temperature, pitch angle distribution of $\sim 272 \mathrm{eV}$ electrons, magnitude, components and $\phi$ angle of magnetic field, and the $\mathrm{O}^{7+} / \mathrm{O}^{6+}$ and $\mathrm{C}^{6+} / \mathrm{C}^{5+}$ charge state ratios of oxygen and carbon. (b) Low density region and HCS crossing observed by ACE (vertical red dashed line). The first panel is the proton density data with 12min resolution from SWICS, the second panel is the proton bulk speed, and the third panel is the $\phi$ angle of magnetic field. The vertical black dashed line and pink region in both $a$ and $b$ are the position of the shock and the low density region before the stream interface shown by the vertical black solid line.

Table 2. Event Two time period we studied for spacecraft STEREO B, Wind, ACE and STEREO A.

\begin{tabular}{|c|c|c|c|}
\hline Spacecraft & Time period studied & Low density region time & Stream interface \\
\hline STEREO B & $\mathrm{N} / \mathrm{A}$ & $\mathrm{N} / \mathrm{A}$ & $\mathrm{N} / \mathrm{A}$ \\
\hline Wind & 06/07 0510-06/08 1910 & 06/07 1859-06/07 2241 & 06/08 0216 \\
\hline $\mathrm{ACE}$ & 06/07 0510-06/08 1910 & 06/07 1849-06/07 2206 & 06/08 0156 \\
\hline STEREO A & N/A & $\mathrm{N} / \mathrm{A}$ & $\mathrm{N} / \mathrm{A}$ \\
\hline
\end{tabular}

Notes. All times are in the format of MM/DD HHMM. 


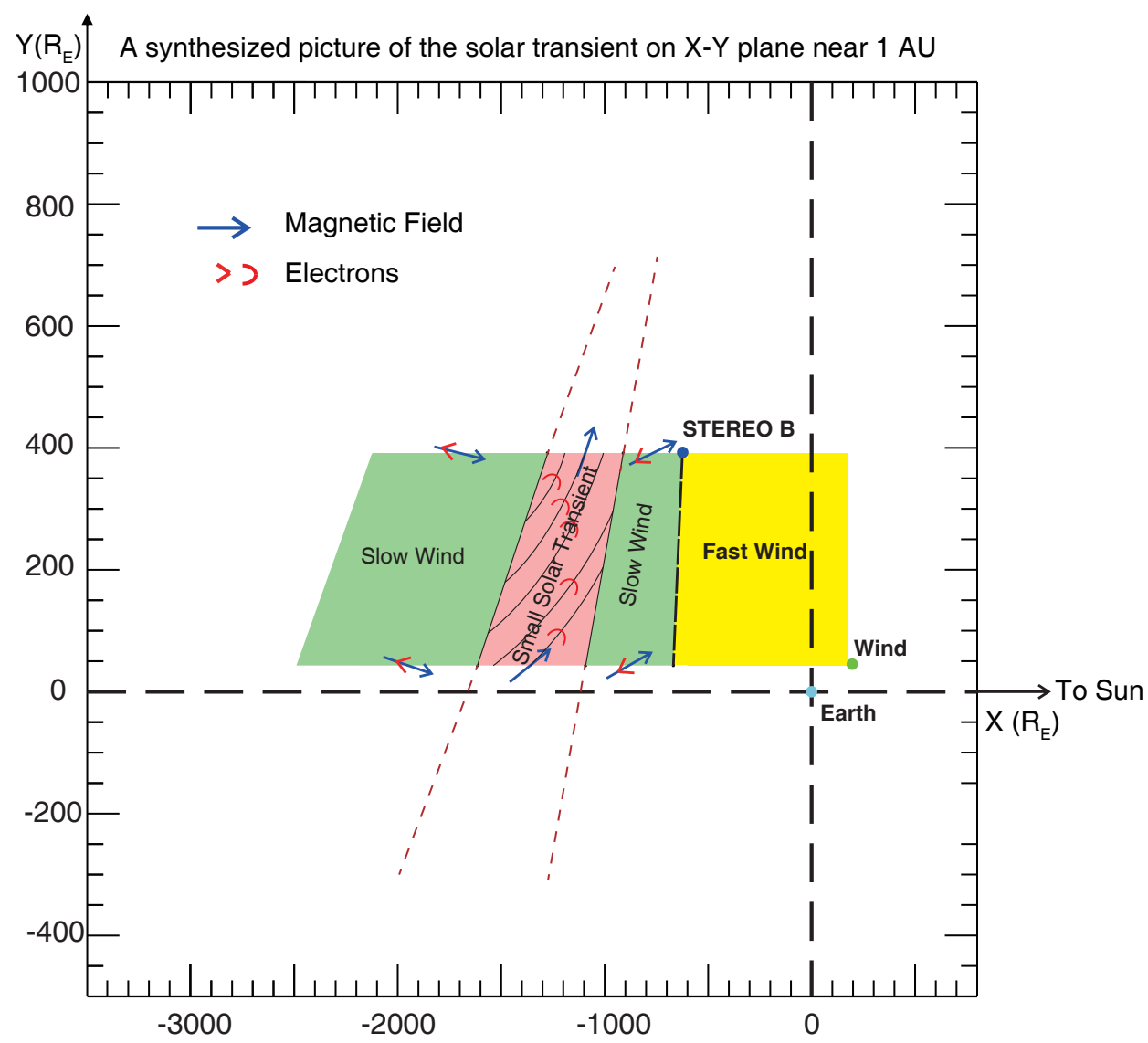

Fig. 8. Synthesized picture of the solar transient near $1 \mathrm{AU}$. The low density area is shown in pink. The surrounding slow wind is in green, while the fast wind is in yellow. The blue arrows represent the directions of magnetic field, the red arrows and red half circles represent the suprathermal electron flows. Semicircles are used for the region where the suprathermal electrons are scattered to a half-sphere distribution. Black lines within the pink region represent the mapping of field lines.
Figures 10a and $\mathrm{b}$ present the spectroscopic plot based on the Extreme ultraviolet Imaging Telescope (EIT; Delaboudinière et al. 1995) on board the SOHO and the Atmospheric Imaging Assembly (AIA; Lemen et al. 2012) on board the Solar Dynamics Observatory (SDO), respectively. The reason we use AIA image for event Two instead EIT is because it has better temporal and spatial resolution than the SOHO EIT image. The observed low density solar wind is traced back to a small coronal hole near the active region marked by a red cross. Here we used the standard two-step mapping procedure to trace the solar wind back to the solar surface (Neugebauer et al. 1998, 2002). Although it has some uncertainties within $10^{\circ}$ according to Neugebauer et al. (2002), this method has been extensively used to study the origin of solar wind (Zhao et al. 2017; Fu et al. 2015, 2017). In event One, the small coronal hole is located at the eastern boundary of the active region, while in event Two it is to the west of the active region. This coronal hole may be the origin of the observed low density plasma with compositional signatures of a coronal hole. The footpoint of event Two is located at the boundary between the small coronal hole and the active region. Usually, the active region is large with bright extended loops. The footpoint appears to overlap with the bright loops, due to a visual effect. Figures $10 \mathrm{c}$ and d are the potentialfield source-surface (PFSS) extrapolations to the observed photospheric field. The green square shows the vicinity of the small transient origin for those events. Figures 10c and d both show that the open field lines are coming from the boundary of closed magnetic field near an active region.

\section{Discussion}

In this section, we discuss the origin of the low density region, considering both coronal hole origin and small scale transient.

\subsection{Coronal hole origin}

For both events, a small parcel plasma of peculiar low proton density and charge states is observed. Could they have an origin associated with coronal holes like the fast solar wind?

Table 3 lists the proton density, temperature, alpha-to-proton number density ratio, and carbon and oxygen charge state ratios of this unusual region, along with typical values for fast and slow solar wind, obtained from Schwenn (2006), von Steiger et al. (2000), Zhao et al. (2014), and Cranmer et al. (2017). The carbon and oxygen charge state ratios were calculated using the freezing-in temperature (i.e., electron temperature corresponding to a given charge-state ratio in coronal equilibrium) from Cranmer et al. (2017) and the ion ionization and recombination rates given by Mazzotta et al. (1998). Except for the speed, the density, proton temperature, composition, and charge-state ratio of the low density region fit in the range of the fast wind for events One and Two.

It has been noted by Lepri et al. (2013) that the compositional parameters vary systematically during the solar cycle and that the charge state ratios for slow solar wind were particularly low during the extended solar minimum of 2008-2009, possibly because of reduced activity and weak solar polar field during this extended solar minimum (Galvin et al. 2009; de Toma 2011). However, during the time period of interest (April 2007) the charge state ratios had not yet reached the very low charge state ratios reported for the 2008-2009 solar minimum time period. Table 4 lists the average charge state ratios for a reference period in 2007 (days 1-180), as derived from two-hour averages measured by ACE/SWICS for slow wind $\left(V_{\mathrm{sw}}<350 \mathrm{~km} \mathrm{~s}^{-1}\right)$ and fast wind $\left(V_{\mathrm{sw}}>550 \mathrm{~km} \mathrm{~s}^{-1}\right)$, together with those for 2008-2009 from (Lepri et al. 2013). It is obvious that for both fast and slow winds, the oxygen charge state ratios during the first half of 2007 


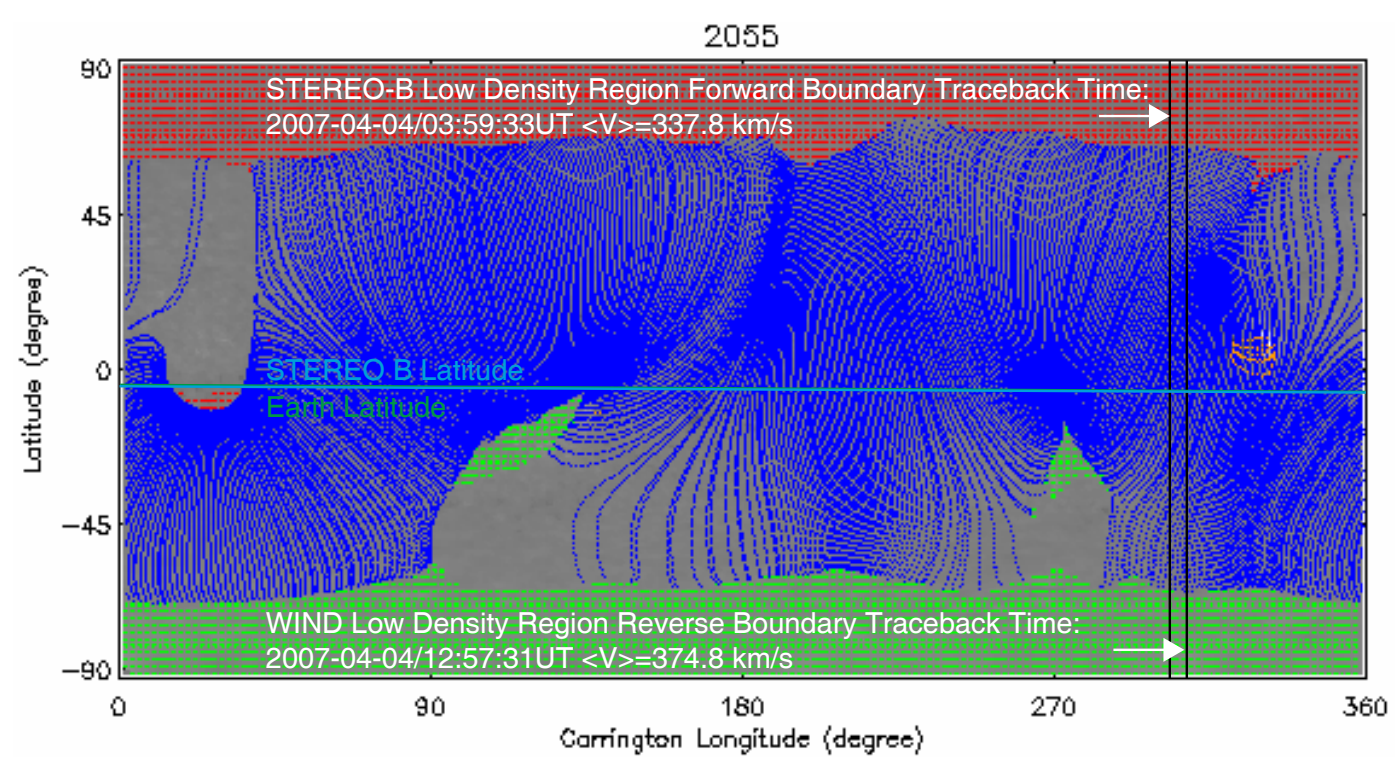

(a)

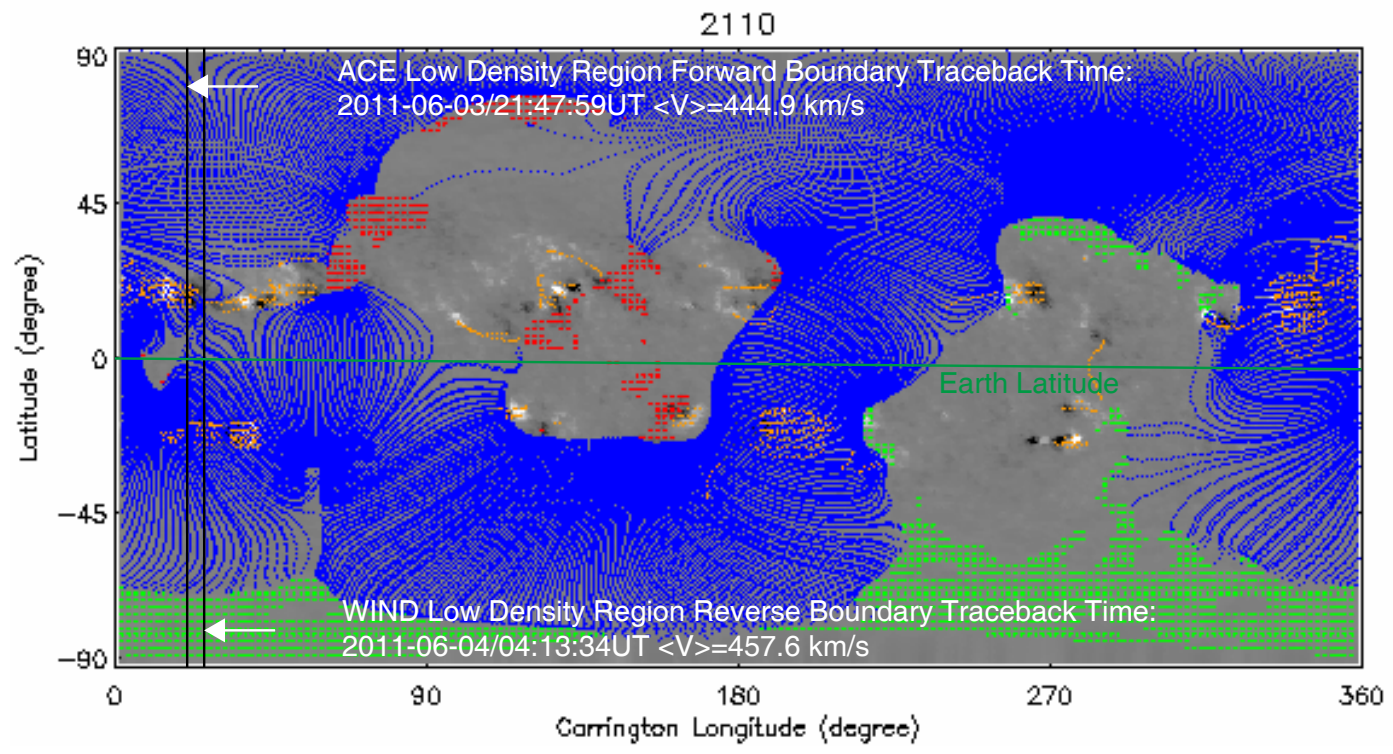

(b)

Fig. 9. (a) Trajectory of STEREO-B and Earth overlaid on the GONG synoptic map of CR 2055. (b) Trajectory of Earth overlaid on the GONG synoptic map of CR 2110. The black solid line shows the possible traced back position of the small coronal hole wind on the synoptic map.

are still as high as the previous solar minimum, unlike those in the year of 2008-2009. Therefore, the lower charge state ratio within the lower density region is unmistakably a fast wind-like feature.

An early statistical study by Zhao et al. (2014) found some low speed solar wind intervals with lower oxygen charge state ratios. A later study by Stakhiv et al. (2015) also suggests that some slow wind has the composition of fast wind, which they call a "boundary wind". However, none of these studies performed a detailed event study including the exact time of the observations. What they discussed may also be explained by a fast wind following the stream interface; their low speed may have resulted from the interaction with the preceding slow wind.

A few models have been proposed to explain the origin of the slow solar wind; a recent one proposed by Antiochos et al. (2007) related its origin with coronal holes at low latitudes. Such coronal holes during solar minimum have been documented by Luhmann et al. (2009) and de Toma (2011). Later studies by
Titov et al. (2011) suggest that such low latitude coronal holes must be connected to polar holes by a dynamic narrow corridor. The S-Web model proposed by Antiochos et al. (2011) suggests that the plasma on closed field lines is sporadically released when the corridor reforms as a result of reconnection (Antiochos et al. 2007, 2011; Titov et al. 2011). The S-web model answers some key questions, such as the wide latitudinal extent of the slow wind, but open questions remain. One question is whether such small coronal holes emit solar wind. If they do, what properties do they have? A recent study by Wang (2017) suggests that slow wind may come out of a coronal hole at lower latitude near an active region. However, their observation was made during the solar maximum when the Sun's magnetic field was disordered and rather large coronal holes may have appeared at low latitudes (see Fig. 16 in Wang 2017). On the other hand, they were using data with rather low time resolution and may have missed some regions restricted to shorter timescales. The small solar wind transient we reported may have originated from a coronal hole. 


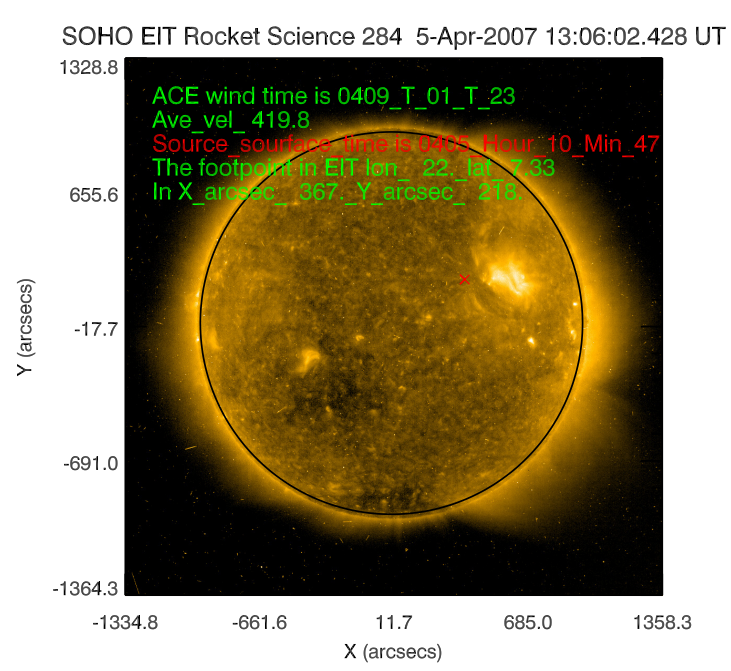

(a)

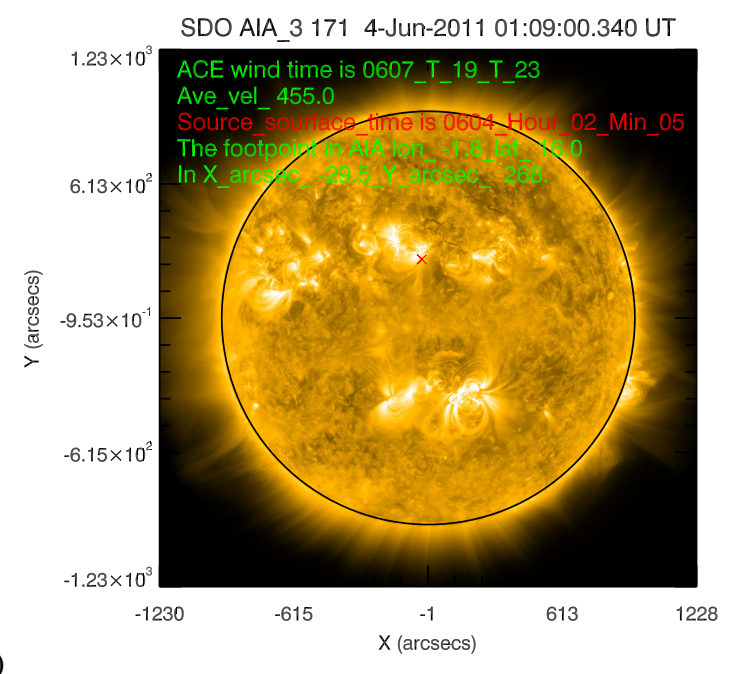

(b)
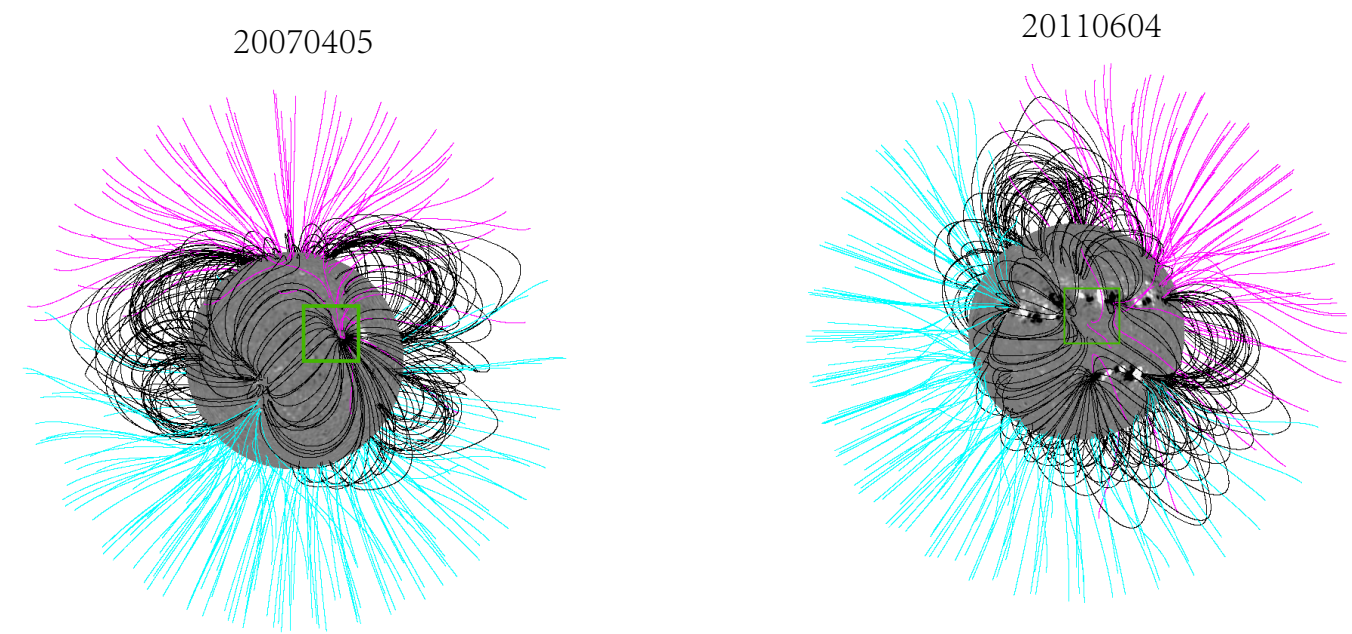

(c)

(d)

Fig. 10. (a) Footpoint of event One observed by ACE traced back on the spectroscopic image taken by EIT $284 \AA$ A, SOHO; (b) Footpoint of event Two observed by ACE traced back on the spectroscopic image taken by AIA 171 A, SDO; $(c)$ PFSS investigation of CR 2055; (d) PFSS investigation of CR 2110. In $c$ and $d$, the open field lines are in cyan (magenta) when negative (positive); the closed field lines are in black. The green square is the PFSS region focused on.

Table 3. Typical parameters in the slow wind, the fast wind, and the special region.

\begin{tabular}{lcccc}
\hline \hline & Slow wind & Fast wind & Event One & Event Two \\
\hline Proton density $\left(\mathrm{cm}^{-3}\right)$ & $5-20$ & $2-4$ & 4.0 & 4.0 \\
Proton temperature $(\mathrm{MK})$ & $0.03-0.1$ & $0.1-0.3$ & $0.1-0.2$ & 0.1 \\
Helium content $(\mathrm{He} / \mathrm{H})$ & $0.5-4 \%$ & $3-5 \%$ & $4-6 \%$ & $2 \%$ \\
$\mathrm{C}^{6+} / \mathrm{C}^{5+}$ & $1.8-4.8$ & $0.24-1.3$ & 0.56 & $0.4-0.7$ \\
$\mathrm{O}^{7+} / \mathrm{O}^{6+}$ & $0.13-0.38$ & $0.01-0.08$ & 0.05 & 0.08 \\
\hline
\end{tabular}

\subsection{Small solar transient}

Small solar wind transients have been thoroughly studied. As suggested in the introduction, most small transients occur in slow solar wind, and they have some characteristics (e.g., enhanced magnetic field strength, low plasma beta, Alfvén Mach number) based on statistical studies (Yu et al. 2014, 2016). Furthermore, the above statistical results clearly show that the signatures of small transients vary greatly (as shown by the large error bars in
Table 4. Charge state ratio $\mathrm{O}^{7+} / \mathrm{O}^{6+}$ in slow wind, and in fast wind for the previous solar minimum (1998-2000), for the year 2007, and for the period 2008-2009.

\begin{tabular}{lcccc}
\hline \hline & \multicolumn{2}{c}{ Slow wind } & \multicolumn{2}{c}{ Fast wind } \\
\cline { 2 - 5 } & Mean & Range & Mean & Range \\
\hline $1998-2000$ & 0.22 & $0.15-0.41$ & 0.040 & $0.02-0.06$ \\
First half of 2007 & 0.20 & $0.13-0.33$ & 0.014 & $0.01-0.03$ \\
$2008-2009$ & 0.06 & $0.03-0.13$ & 0.013 & $0.01-0.03$ \\
\hline
\end{tabular}

Figs. 3-5 in Yu et al. 2016) even in the same year, implying that small transients may originate from different source regions and formation mechanisms (Janvier et al. 2014; Huang et al. 2017). Here we compare our events with the typical signatures of small transients. Table 5 lists magnetic field strength, Alfvén Mach number, plasma $\beta$, proton $\beta$, and the ratio of proton temperature to the expected temperature for both events and for the average values for small solar wind transients observed by Wind in 2007 (Yu et al. 2016). 
Table 5. Average magnetic field, the average Mach number, plasma $\beta$, proton $\beta$, and the ratio of proton temperature vs the expected temperature for the low density region and the solar transient observed by Wind in 2007.

\begin{tabular}{lccc}
\hline \hline & Event One & Event Two & Solar wind transient \\
\hline Magnetic field magnitude & 14.8 & 9.1 & 9.10 \\
Mach number & 2.9 & 4.9 & 6.34 \\
Plasma $\beta$ & $0.1-0.3$ & 0.38 & 1.23 \\
Proton $\beta$ & 0.12 & 0.13 & 0.36 \\
$T_{\mathrm{p}} / T_{\exp }$ & 9.6 & 0.87 & 1.74 \\
\hline
\end{tabular}

It is obvious that both events have significantly smaller plasma and proton $\beta$, and lower Alfvén Mach number. The comparison of other parameters with the average of solar wind transient is quite different for these two events: the magnetic field magnitude is higher for event One but similar for event Two; $T_{\mathrm{p}} / T_{\exp }$ is much higher for event One but lower for event Two. Therefore, these prominent differences in our events suggest that they may have a different origin from most of other solar wind transients.

\section{Conclusion and future work}

We report two events of low density solar transients in a compressed slow wind from STEREO B, Wind, and ACE observations. These two events are representative and were selected from a few similar events. Within the region the charge states of carbon and oxygen, the elemental composition $(\mathrm{He} / \mathrm{H})$, and the lower proton density suggest that these solar transients have similar characteristics to those of fast wind originating from a coronal hole. For both events we trace back to the surface of the Sun and find they are connected to the vicinity of a small coronal hole near an active region at low latitudes. Event One has significantly different characteristic parameters, such as magnetic field magnitude, proton $\beta$, and the ratio of proton temperature to the expected temperature from most other solar wind transients; event Two has a very typical magnetic field, but the other parameters are quite different.

Therefore, we draw the following conclusions:

1. Some small solar wind transients observed in slow wind may originate from small coronal holes at lower latitudes.

2 . These coronal hole solar transients have very different temperature and proton $\beta$ from other solar transients, consistent with their origin.

Bruno \& Telloni (2015) studied the Alfvénicity of the slow wind, suggesting that slow wind may be classified as one of two types. The first type (slow wind with lower degree of Alfvénicity) originates from the active regions or streamer belt; the second type originates from the coronal hole boundary, and therefore has higher Alfvénicity. A recent study by D'Amicis et al. (2019) showed that Alfvénic slow winds share other common characteristics with fast winds, supporting the idea that these slow winds may originate from solar coronal holes. Our study showed that some solar wind transients within the first type of slow wind may also originate from small coronal holes. A recent study by Ko et al. (2018) also suggest that the slow wind may have very different origin based on their velocity fluctuations.

There are questions that remain unanswered. First, what does such a flow out of small coronal holes mean for the slow wind originating model? A schematic model is illustrated in Fig. 11. A low latitude corona hole is connected to a major polar hole by a narrow corridor, and fast wind, slow wind, and fast wind-like

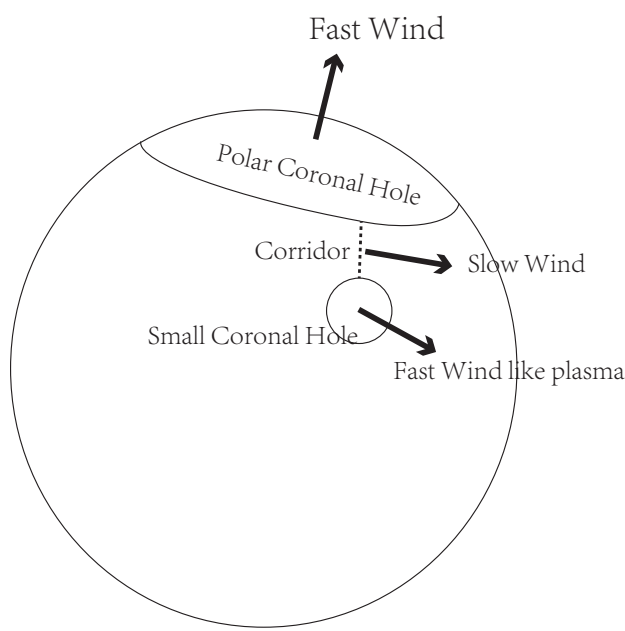

Fig. 11. Schematic plot showing small coronal hole, slow wind, and fast wind origin.

plasma flowing out of them separately. Why are the solar winds coming out of the corridor and the small coronal hole different even though they are both located in the streamer belt region? A possible answer could be the following: the topological stable corridor requires, in response to local photospheric changes, dynamic adjustments of the magnetic field configuration that lead to the release of closed-field plasma with slow solar wind characteristics at the quasi-separatrix layers, as described by the S-web model (Antiochos et al. 2011). The small coronal hole is quite stable, and plasma outflows have a composition similar to that of outflows from the polar hole. The time span of the solar transient is determined by the lifetime of the small coronal hole.

Second, how do they interact with the active region in the vicinity? For event One, the distribution of the suprathermal electrons suggests that that field lines are rolled back from its original configuration. How does this happen? Another intriguing phenomenon, the coincidence of heliospheric current sheet and the stream interface, was observed by STEREO A (Huang et al. 2016a,b). The coincidence was explained by interchange reconnection of open field lines with closed field lines. Is this coincidence related to the low density solar transient with very atypical proton $\beta$ and other features? Answering these questions may further our understanding of the origin of slow wind. The Parker Solar Probe (Bale et al. 2016; Fox et al. 2016; Kasper et al. 2016) and future missions like Solar Orbiter (Müller \& Marsden 2013) will provide useful information to answer these questions.

Acknowledgements. This work is supported by the Chinese Academy of Science, the Chinese National Science Foundation of contract number 41674173 and 41904152, the Beijing Natural Science Foundation (8194076) and the Specialized Research Fund for State Key Laboratory of China. We also thank GONG for the synoptic coronal hole plots and SolarSoft library for the PFSS model. The in situ data are provided by the STEREO (http://stereo-ssc . nascom.nasa.gov), Wind (http://pwg.gsfc.nasa.gov/wind.shtml) and ACE (http://www.srl.caltech.edu/ACE/ASC/) Science Centers. We also benefit from discussions with Jiansen He from Peking University.

\section{References}

Abbo, L., Ofman, L., Antiochos, S. K., et al. 2016, Space Sci. Rev., 201, 55 Acuña, M. H., Curtis, D., Scheifele, J. L., et al. 2008, Space Sci. Rev., 136, 203 Antiochos, S. K., DeVore, C. R., Karpen, J. T., \& Mikić, Z. 2007, ApJ, 671, 936 Antiochos, S. K., Mikić, Z., Titov, V. S., Lionello, R., \& Linker, J. A. 2011, ApJ, 731,112 
Bale, S. D., Goetz, K., Harvey, P. R., et al. 2016, Space Sci. Rev., 204, 49

Bame, S. J., Goldstein, B. E., Gosling, J. T., et al. 1993, Geophys. Res. Lett., 20, 2323

Borovsky, J. E. 2010, J. Geophys. Res. (Space Physics), 115, A09101

Borrini, G., Gosling, J. T., Bame, S. J., Feldman, W. C., \& Wilcox, J. M. 1981, J. Geophys. Res., 86, 4565

Broiles, T. W., Desai, M. I., \& McComas, D. J. 2012, J. Geophys. Res. (Space Physics), 117, A03102

Bruno, R., \& Telloni, D. 2015, ApJ, 811, L17

Burlaga, L. F., Scudder, J. D., Klein, L. W., \& Isenberg, P. A. 1990, J. Geophys. Res., 95, 2229

Cranmer, S. R., Gibson, S. E., \& Riley, P. 2017, Space Sci. Rev., 212, 1345

Crooker, N. U., Gosling, J. T., Bothmer, V., et al. 1999, Space Sci. Rev., 89, 179

Crooker, N. U., Kahler, S. W., Larson, D. E., \& Lin, R. P. 2004, J. Geophys. Res. (Space Physics), 109, A03108

D’Amicis, R., Matteini, L., \& Bruno, R. 2019, MNRAS, 483, 4665

de Toma, G. 2011, Sol. Phys., 274, 195

Delaboudinière, J. P., Artzner, G. E., Brunaud, J., et al. 1995, Sol. Phys., 162, 291

Elliott, H. A., McComas, D. J., Schwadron, N. A., et al. 2005, J. Geophys. Res. (Space Physics), 110, A04103

Feldman, U., Landi, E., \& Schwadron, N. A. 2005, J. Geophys. Res. (Space Physics), 110, A07109

Foullon, C., Lavraud, B., Wardle, N. C., et al. 2009, Sol. Phys., 259, 389

Fox, N. J., Velli, M. C., Bale, S. D., et al. 2016, Space Sci. Rev., 204, 7

Fu, H., Li, B., Li, X., et al. 2015, Sol. Phys., 290, 1399

Fu, H., Madjarska, M. S., Xia, L., et al. 2017, ApJ, 836, 169

Galvin, A. B., Kistler, L. M., Popecki, M. A., et al. 2008, Space Sci. Rev., 136, 437

Galvin, A. B., Popecki, M. A., Simunac, K. D. C., et al. 2009, Ann. Geophys., 27,3909

Geiss, J., Gloeckler, G., \& von Steiger, R. 1995, Space Sci. Rev., 72, 49

Gloeckler, G., Geiss, J., Balsiger, H., et al. 1992, A\&AS, 92, 267

Gosling, J. T. 1996, ARA\&A, 34, 35

Gosling, J. T., Asbridge, J. R., Bame, S. J., \& Feldman, W. C. 1978, J. Geophys. Res., 83, 1401

Gosling, J. T., Bame, S. J., McComas, D. J., et al. 1993, Geophys. Res. Lett., 20, 2789

He, J., Marsch, E., Tu, C., \& Tian, H. 2009, ApJ, 705, L217

Huang, J., Liu, Y. C. M., Klecker, B., \& Chen, Y. 2016a, J. Geophys. Res. (Space Physics), 121, 19

Huang, J., Liu, Y. C. M., Qi, Z., et al. 2016b, J. Geophys. Res. (Space Physics), 121,768

Huang, J., Liu, Y. C. M., Peng, J., et al. 2017, J. Geophys. Res. (Space Physics), 122,6927

Huang, J., Liu, Y. C. M., Peng, J., et al. 2018, J. Geophys. Res. (Space Physics), 123,7167

Janvier, M., Démoulin, P., \& Dasso, S. 2014, Sol. Phys., 289, 2633

Jian, L., Russell, C. T., Luhmann, J. G., \& Skoug, R. M. 2006, Sol. Phys., 239, 337

Jian, L. K., Luhmann, J. G., Russell, C. T., \& Galvin, A. B. 2019, Sol. Phys., 294, 31

Kahler, S. W., Crooker, N. U., \& Gosling, J. T. 1996, J. Geophys. Res., 101, 24373

Kaiser, M. L., Kucera, T. A., Davila, J. M., et al. 2008, Space Sci. Rev., 136, 5

Kasper, J. C., Abiad, R., Austin, G., et al. 2016, Space Sci. Rev., 204, 131

Kilpua, E. K. J., Luhmann, J. G., Gosling, J., et al. 2009, Sol. Phys., 256, 327

Ko, Y.-K., Roberts, D. A., \& Lepri, S. T. 2018, ApJ, 864, 139

Lavraud, B., Opitz, A., Gosling, J. T., et al. 2010, Ann. Geophys., 28, 233

Lemen, J. R., Title, A. M., Akin, D. J., et al. 2012, Sol. Phys., 275, 17

Lepping, R. P., Acũna, M. H., Burlaga, L. F., et al. 1995, Space Sci. Rev., 71, 207
Lepri, S. T., Landi, E., \& Zurbuchen, T. H. 2013, ApJ, 768, 94

Lin, R. P., Anderson, K. A., Ashford, S., et al. 1995, Space Sci. Rev., 71, 125

Liu, Y. C. M., Huang, J., Wang, C., et al. 2014, J. Geophys. Res. (Space Physics), 119,8721

Lopez, R. E., \& Freeman, J. W. 1986, J. Geophys. Res., 91, 1701

Luhmann, J. G., Curtis, D. W., Schroeder, P., et al. 2008, Space Sci. Rev., 136, 117

Luhmann, J. G., Lee, C. O., Li, Y., et al. 2009, Sol. Phys., 256, 285

Mazzotta, P., Mazzitelli, G., Colafrancesco, S., \& Vittorio, N. 1998, A\&AS, 133, 403

McComas, D. J., Bame, S. J., Barker, P., et al. 1998, Space Sci. Rev., 86, 563

McComas, D. J., Barraclough, B. L., Funsten, H. O., et al. 2000, J. Geophys. Res., 105, 10419

McComas, D. J., Elliott, H. A., Gosling, J. T., et al. 2002, Geophys. Res. Lett., 29, 1290

Moldwin, M. B., Ford, S., Lepping, R., Slavin, J., \& Szabo, A. 2000, Geophys. Res. Lett., 27, 57

Müller, D., Marsden, R. G., \& St. Cyr, O. C., \& Gilbert, H. R., 2013, Sol. Phys., 285,25

Neugebauer, M., Forsyth, R. J., Galvin, A. B., et al. 1998, J. Geophys. Res., 103 14587

Neugebauer, M., Liewer, P. C., Smith, E. J., Skoug, R. M., \& Zurbuchen, T. H. 2002, J. Geophys. Res. (Space Physics), 107, 1488

Neugebauer, M., Steinberg, J. T., Tokar, R. L., et al. 2003, Space Sci. Rev., 105, 661

Ogilvie, K. W., Chornay, D. J., Fritzenreiter, R. J., et al. 1995, Space Sci. Rev., 71,55

Owens, M. J., Crooker, N. U., \& Lockwood, M. 2013, J. Geophys. Res. (Space Physics), 118, 1868

Parker, E. N. 1958, ApJ, 128, 664

Rouillard, A. P., Savani, N. P., Davies, J. A., et al. 2009, Sol. Phys., 256, 307

Sauvaud, J. A., Larson, D., Aoustin, C., et al. 2008, Space Sci. Rev., 136, 227

Schwenn, R. 2006, Space Sci. Rev., 124, 51

Simunac, K. D. C., Kistler, L. M., Galvin, A. B., et al. 2009, Sol. Phys., 259, 323

Smith, C. W., L'Heureux, J., Ness, N. F., et al. 1998, Space Sci. Rev., 86, 613

Stakhiv, M., Landi, E., Lepri, S. T., Oran, R., \& Zurbuchen, T. H. 2015, ApJ, 801,100

Stone, E. C., Frandsen, A. M., Mewaldt, R. A., et al. 1998, Space Sci. Rev., 86,

Titov, V. S., Mikić, Z., Linker, J. A., Lionello, R., \& Antiochos, S. K. 2011, ApJ, 731,111

von Steiger, R., Schwadron, N. A., Fisk, L. A., et al. 2000, J. Geophys. Res., 105, 27217

Wang, X., Zhao, L., Tu, C., \& He, J. 2019, ApJ, 871, 204

Wang, Y. M. 2016, ApJ, 833, 121

Wang, Y. M. 2017, ApJ, 841, 94

Wimmer-Schweingruber, R. F., von Steiger, R., \& Paerli, R. 1997, J. Geophys. Res., 102, 17407

Xu, F., \& Borovsky, J. E. 2015, J. Geophys. Res. (Space Physics), 120, 70

Yu, W., Farrugia, C. J., Lugaz, N., et al. 2014, J. Geophys. Res. (Space Physics), 119,689

Yu, W., Farrugia, C. J., Galvin, A. B., et al. 2016, J. Geophys. Res. (Space Physics), 121, 5005

Zhao, L., Zurbuchen, T. H., \& Fisk, L. A. 2009, Geophys. Res. Lett., 36, L14104 Zhao, L., Landi, E., Zurbuchen, T. H., Fisk, L. A., \& Lepri, S. T. 2014, ApJ, 793, 44

Zhao, L., Landi, E., Lepri, S. T., et al. 2017, ApJ, 846, 135

Zirker, J. B. 1977, Rev. Geophys. Space Phys., 15, 257

Zurbuchen, T. H., von Steiger, R., Gruesbeck, J., et al. 2012, Space Sci. Rev., 172, 41 